\title{
Intra-Species Interactions in Streptococcus pneumoniae Biofilms
}

\author{
Carina Valente ${ }^{1 \neq}$, Ana R. Cruz ${ }^{1 \dagger \neq}$, Adriano O. Henriques ${ }^{2}$ and Raquel Sá-Leão ${ }^{1 *}$ \\ ${ }^{1}$ Laboratory of Molecular Microbiology of Human Pathogens, Instituto de Tecnologia Química e Biológica António Xavier, \\ Universidade Nova de Lisboa, Oeiras, Portugal, ${ }^{2}$ Laboratory of Microbial Development, Instituto de Tecnologia Química e \\ Biológica António Xavier, Oeiras, Portugal
}

\section{OPEN ACCESS}

Edited by:

Guangchun Bai,

Albany Medical College, United States

Reviewed by:

Muhammad Ammar Zafar,

Wake Forest School of Medicine,

United States

Masaya Yamaguchi,

Osaka University, Japan

Lance Edward Keller,

University of Mississippi Medical

Center, United States

*Correspondence:

Raquel Sá-Leão

rsaleao@itqb.unl.pt

${ }^{\dagger}$ Present address:

Ana R. Cruz Laboratory of HostMicrobiota Interaction, Institut Necker

Enfants Malades, Paris, France

${ }^{*}$ These authors have contributed equally to this work and share first authorship

Specialty section:

This article was submitted to

Molecular Bacterial Pathogenesis,

a section of the journal

Frontiers in Cellular and

Infection Microbiology

Received: 27 October 2021

Accepted: 14 December 2021

Published: 05 January 2022

Citation:

Valente $C$, Cruz $A R$, Henriques $A O$ and

Sá-Leão R (2022) Intra-Species

Interactions in Streptococcus

pneumoniae Biofilms.

Front. Cell. Infect. Microbiol. 11:803286.

doi: 10.3389/fcimb.2021.803286
Streptococcus pneumoniae is a human pathogen responsible for high morbidity and mortality worldwide. Disease is incidental and is preceded by asymptomatic nasopharyngeal colonization in the form of biofilms. Simultaneous colonization by multiple pneumococcal strains is frequent but remains poorly characterized. Previous studies, using mostly laboratory strains, showed that pneumococcal strains can reciprocally affect each other's colonization ability. Here, we aimed at developing a strategy to investigate pneumococcal intra-species interactions occurring in biofilms. A 72h abiotic biofilm model mimicking long-term colonization was applied to study eight pneumococcal strains encompassing 6 capsular types and 7 multilocus sequence types. Strains were labeled with GFP or RFP, generating two fluorescent variants for each. Intraspecies interactions were evaluated in dual-strain biofilms (1:1 ratio) using flow cytometry. Confocal microscopy was used to image representative biofilms. Twenty-eight dual-strain combinations were tested. Interactions of commensalism, competition, amensalism and neutralism were identified. The outcome of an interaction was independent of the capsular and sequence type of the strains involved. Confocal imaging of biofilms confirmed the positive, negative and neutral effects that pneumococci can exert on each other. In conclusion, we developed an experimental approach that successfully discriminates pneumococcal strains growing in mixed biofilms, which enables the identification of intra-species interactions. Several types of interactions occur among pneumococci. These observations are a starting point to study the mechanisms underlying those interactions.

Keywords: biofilms, co-colonization, colonization, multiple carriage, competition, intraspecies interactions, Streptococcus pneumoniae

\section{INTRODUCTION}

Streptococcus pneumoniae is an important bacterial pathogen associated with high morbidity and mortality worldwide (GBD, 2018). Nevertheless, disease is a rare event compared with the frequency of asymptomatic nasopharyngeal colonization (Weiser et al., 2018).

Nasopharyngeal colonization is particularly frequent in young children (Gray et al., 1980), where multiple pneumococcal strains can simultaneously colonize the same host, a phenomenon known as multiple serotype carriage or co-colonization (Turner et al., 2011; Valente et al., 2012; Wyllie et al., 
2014; Kamng'ona et al., 2015; Kandasamy et al., 2015; Valente et al., 2016b). Co-colonization is frequent, reaching up to $40 \%$ or $50 \%$, depending on the geographical setting and the methodology used for its detection (Turner et al., 2011; Valente et al., 2012; Wyllie et al., 2014; Kamng'ona et al., 2015; Kandasamy et al., 2015; Valente et al., 2016b).

During colonization, pneumococci form biofilms that confer protection from the host and enable horizontal gene transfer, the main evolutionary mechanism of this species (Barnes et al., 1995; Hiller et al., 2010; Wei and Havarstein, 2012).

Several lines of evidence indicate that intra-species competition (i.e., competition between pneumococcal strains) occurs during cocolonization and has a determinant role in shaping its epidemiology and population structure. For example, the dominance of a limited subset of capsular types (typically 5-6 types) in carriage, when several are known to circulate in a population, is a clear indication that strains have different competitive abilities to colonize (Hausdorff et al., 2005). Introduction of pneumococcal conjugate vaccines worldwide also indicates that, following serotype replacement, the population structure of pneumococci is reshaped due to expansion of a limited number of non-vaccine types in carriage and disease (Moore et al., 2015; Waight et al., 2015; Azarian et al., 2020). In addition, using a mouse model of colonization, Lipsitch and co-workers showed that the presence of a resident pneumococcal strain could affect colonization by a second (challenger) strain (Lipsitch et al., 2000).

The mechanisms mediating pneumococcal intra-species competition have been addressed in a few subsequent studies. Trzciński et al. tested clinical isolates and isogenic variants of six capsular types in a mouse model of multiple-serotype carriage and observed that the competitive ability of a strain to colonize depends strongly, but not exclusively, on the composition of its polysaccharide capsule (Trzciński et al., 2015). Apart from the capsule, the blp (bacteriocin-like peptide) locus and the competence system were also implicated in competitive interactions. Dawid et al., used a 19A clinical isolate and its blp bacteriocin deleted derivative, to demonstrate that the $b l p$ locus promotes intra-species competition, both in vitro (using overlay assays) and in vivo (using a murine model of colonization) (Dawid et al., 2007). In a follow-up study, Wholey et al. observed a cross-stimulation between the blp and competence systems with implications for intra-species interactions (Wholey et al., 2016). Moreover, in dual-strain biofilms, bacteriocin producing pneumococci, not only had a competitive advantage over non-producers, but also increased DNA exchange through natural transformation (Wholey et al., 2016). Using an infant mouse model of colonization, Shen et al. showed that the competitive advantage of a resident strain is quorum sensingdependent and is mediated by the late-competence activated fratricide effectors CbpD and CibAB (Shen et al., 2019). Finally, $\mathrm{Wu}$ et al. tested three clinical isolates, of serotypes $6 \mathrm{~B}, 19 \mathrm{~F}$ and $23 \mathrm{~F}$, in an eight-hour dual-strain biofilm model and showed that strain $19 \mathrm{~F}$ was outcompeted by strains $6 \mathrm{~B}$ and $23 \mathrm{~F}$ through a contact-dependent unknown mechanism (Wu et al., 2017).

While other types of interactions, beyond competition, are theoretically possible among pneumococci, they have remained mostly undescribed. One exception are the studies of Trzciński et al. and Wu et al., who described strain combinations where coexistence was observed suggesting a neutral interaction (Trzciński et al., 2015; Wu et al., 2017). In addition, while using isogenic laboratory strains (D39 wt and D39 pspA/pspC null mutant) to study natural transformation in multi-strain biofilms, Marks and co-authors found that mixed populations of pneumococci may increase the adaptive potential of less fit strains (Marks et al., 2012), suggesting that commensalism may occur between pneumococci.

Despite significant advances on the study of pneumococcal co-colonization during the last two decades, progress has been hampered by methodological constraints associated with the need to quantitatively distinguish cells of different strains when in mixed-strain experimental conditions. While it is recognized that there is significant within strain genetic diversity in pneumococci, the number of strains being used in such studies has been limited and has mainly relied on the use of laboratory strains and its derivatives.

In this study, we developed a strategy to study pneumococci in multi-strain biofilms and applied it to a diverse set of strains which included natural isolates obtained from samples collected from colonized human subjects. We show that various types of intra-species interactions, including commensalism, do occur and can be quantitatively characterized.

\section{MATERIALS AND METHODS}

\section{Study Collection}

The study collection consisted of 12 strains previously isolated from nasopharyngeal samples of healthy children in which cocolonization was detected (Sá-Leão et al., 2009; Valente et al., 2012; Nunes et al., 2016; Valente et al., 2016a; Valente et al., 2016b; Félix et al., 2021) and two laboratory strains, D39 and TIGR4, commonly used in pneumococcal studies worldwide. Epidemiological relevance of selected strains and their serotypes and genotypes are indicated in Tables 1, 2. Four strains were non-encapsulated and were designated as non-typable (NT).

\section{Fluorescent Labelling of S. pneumoniae Strains}

Each strain was labelled with GFP or RFP generating two fluorescently labelled strains. Constructs $\mathrm{P}_{h l p A}-h l p A-g f p \_C a m^{\mathrm{r}}$ (for GFP-labelling) and $\mathrm{P}_{h l p} A^{-} h l p A \_h l p A-r f p \_C a m^{\mathrm{r}}$ (for RFPlabelling), kindly provided by Jan-Willem Veening, were used (Kjos et al., 2015). These constructs bear a translational fusion of the histone-like protein A gene ( $h l p A)$ to GFP or RFP, where the fusion proteins are produced under the control of the $h l p A$ promoter; the constructs also carry a transcriptional fusion to a chloramphenicol resistance gene. Constructs were first integrated by allelic replacement in the genome of S. pneumoniae D39 (laboratory strain) generating strains D39_GFP and D39_RFP. DNA was extracted from either D39_GFP or D39_RFP and 
TABLE 1 | Rationale for selection of S. pneumoniae strains based on epidemiological relevance.

\begin{tabular}{|c|c|c|c|}
\hline Serotype & $\begin{array}{l}\text { PCV13 } \\
\text { serotype }\end{array}$ & Observations & References \\
\hline 3 & Yes & $\begin{array}{l}\text { High invasive disease potential } \\
\text { Increasing in PCV13 era }\end{array}$ & (Sá-Leão et al., 2011; Horácio et al., 2016) \\
\hline $15 \mathrm{~A}$ & No & Increasing in carriage in PCV13 era & (Félix et al., 2021) \\
\hline $19 \mathrm{~A}$ & Yes & Highly prevalent in carriage and disease before the introduction of PCV13 & (Isturiz et al., 2017) \\
\hline $19 F$ & Yes & Remains in carriage in PCV13 era & (Nunes et al., 2016; Félix et al., 2021) \\
\hline $22 F ; 33 F$ & No & $\begin{array}{l}\text { Increasing in carriage in PCV13 era } \\
\text { Important cause of invasive pneumococcal disease } \\
\text { Targeted by upcoming PCV15 and PCV20 vaccines }\end{array}$ & (Moore et al., 2015; Félix et al., 2021) \\
\hline NT & No & $\begin{array}{l}\text { Non-encapsulated } \\
\text { Very frequent in colonization } \\
\text { Frequently detected in co-colonization } \\
\text { Associated with multidrug resistance } \\
\text { Associated to conjunctivitis outbreaks }\end{array}$ & (Marks et al., 2012; Valentino et al., 2014; Valente et al., 2016b) \\
\hline
\end{tabular}

PCV13, PCV15 and PCV20 correspond to 13-valent, 15-valent and 20-valent pneumococcal conjugate vaccines. NT - non-typeable.

amplified by PCR using primers previously described (Kjos et al., 2015). PCR products were purified with Exo-SAP and used for transformation of other strains.

Two transformation protocols, in liquid medium and in biofilms, were used. For transformation in liquid medium cells were grown in $\mathrm{C}+\mathrm{Y}$ ( $\mathrm{pH}$ 7.4) (Junges et al., 2017) to an optical density of 0.5 at $600 \mathrm{~nm}\left(\mathrm{OD}_{600}\right)$, diluted at a $1: 100$ ratio and grown until an $\mathrm{OD}_{600 \mathrm{~nm}}$ of 0.1 was reached; at this time, 100ng/ $\mathrm{mL}$ of CSP1 (NH2-EMRLSKFFRDFILQRKK-COOH) or CSP2 (NH2-EMRISRIILDFLFLRKK-COOH) (Mimotopes, Australia) and $400 \mathrm{ng} / \mathrm{mL}$ of DNA were added. The mixture was incubated for $3 \mathrm{~h}$ at $37^{\circ} \mathrm{C}$.

For clinical isolates which did not yield transformants using the conditions described above, transformation in biofilms was attempted. Cells were grown in $\mathrm{C}+\mathrm{Y}$ ( $\mathrm{pH}$ 7.4) (Junges et al., 2017) to an $\mathrm{OD}_{600}$ of 0.5 , diluted at a 1:100 ratio, grown until an $\mathrm{OD}_{600}$ of 0.2 and transferred to 24 -well plates (Costar, Corning), in a final volume of $2 \mathrm{~mL}$ per well. Plates were incubated for $4 \mathrm{~h}$ at $34^{\circ} \mathrm{C}$ in a $5 \% \mathrm{CO}_{2}$ atmosphere to promote biofilm formation (Wei and Havarstein, 2012). The supernatant was carefully aspirated to remove planktonic bacteria and replaced with $2 \mathrm{~mL}$ of fresh medium supplemented with $0.5 \mu \mathrm{g} / \mathrm{mL}$ of DNA and the appropriate CSP at a final concentration of either $100 \mathrm{ng} / \mathrm{mL}$ or $1000 \mathrm{ng} / \mathrm{mL}$.

In both protocols transformants were selected on TSA blood plates supplemented with $4 \mu \mathrm{g} / \mathrm{mL}$ chloramphenicol. For selected clones, the presence of the fusion constructs at the hlpA locus was first verified by PCR.

\section{Fluorescence Microscopy}

For selected clones resulting from the integration of the $\mathrm{P}_{h l p A^{-}}$ $h l p A-g f p$ or $\mathrm{P}_{h l p A}-h l p A \_h l p A-r f p$ fusions, production of GFP or RFP was assessed by fluorescence microscopy. Two $\mathrm{mL}$ of exponential phase culture were centrifuged at $5900 \mathrm{~g}$ for $10 \mathrm{~min}$ and pellets were resuspended in PBS. Cell suspensions were mounted on a slide covered with a thin layer of $1.7 \%$ agarose. Images were acquired on a Leica DM 6000B fluorescence microscope controlled by MetaMorph V5.8 software through a 100X 1.4 NA oil immersion objective and captured with an Andor iXon 885 EMCCD camera. Filters for image acquisition were set for contrast phase optics, TX2 (excitation: 560/40nm; emission: 645/75nm), and FITC (excitation: 480/40 nm; emission: 527/30nm). Exposure times were $50 \mathrm{~ms}$ for contrast phase, $3000 \mathrm{~ms}$ for TX2, and 500ms for FITC.

TABLE 2 | Bacterial strains initially chosen for the study of intra-species interactions.

\begin{tabular}{|c|c|c|c|c|}
\hline Strain & Serotype & MLST $^{1}$ & Observation $^{2}$ & Reference \\
\hline D39 & 2 & 595 & selected & (Avery et al., 1944) \\
\hline TIGR4 & 4 & 205 & selected & (Tettelin et al., 2001) \\
\hline 2099 & 3 & 260 & - & (Nunes et al., 2016) \\
\hline 7632 & $15 \mathrm{~A}$ & 8322 & selected & (Nunes et al., 2016) \\
\hline 5262.1 & $19 A$ & 276 & - & (Sá-Leão et al., 2009) \\
\hline 5902 & $19 A$ & 63 & - & (Simões et al., 2011) \\
\hline 1990 & $19 F$ & 177 & selected & (Nunes et al., 2016) \\
\hline 5756 & $22 \mathrm{~F}$ & 433 & selected & (Sá-Leão et al., 2009) \\
\hline 8046 & $22 \mathrm{~F}$ & 9161 & - & (Nunes et al., 2016) \\
\hline 8276 & $33 F$ & 717 & - & (Félix et al., 2021) \\
\hline 5262.2 & NT & 344 & - & (Sá-Leão et al., 2009) \\
\hline 5435 & NT & 344 & selected & (Sá-Leão et al., 2009) \\
\hline 7031 & NT & 344 & selected & (Nunes et al., 2016) \\
\hline 6209 & NT & 4583 & selected & (Simões et al., 2011) \\
\hline
\end{tabular}

MLST, multilocus sequence typing; NT, non-typeable (non-encapsulated). ${ }^{1}$ STs mainly associated with colonization isolates (www.pubm/st.org).

2Strains tested in dual-strain biofilms are indicated as "selected". Other stains did not meet one or more validation criteria and were excluded. 


\section{Biofilm Growth Medium for the Study of Intra-Species Interactions}

Strains were grown in a modified version of the chemically defined medium described by van de Rijn and Kessler, herein named $\mathrm{mCDM}$, in which the final concentration of cysteine- $\mathrm{HCl}$ was increased to $0.8 \mathrm{mg} / \mathrm{mL}$ (instead of $0.5 \mathrm{mg} / \mathrm{mL}$ ), choline chloride was added at a final concentration of $1 \mathrm{mg} / \mathrm{mL}$, except when otherwise indicated. The carbon source was galactose $(10 \mathrm{mg} / \mathrm{mL})$ instead of glucose (the complete recipe of $\mathrm{mCDM}$ is given in Table S1). Cysteine promotes faster bacterial growth as it can act as a radical scavenger and as an additional amino-acid source. Choline is a nutritional requirement for pneumococcal growth and was added at a concentration of $1 \mathrm{mg} / \mathrm{mL}$ to promote better growth (Rane and Subbarow, 1940; Tomasz, 1967). Galactose was chosen as it is the most abundant sugar in the human nasopharynx (Blanchette et al., 2016). All experiments were carried out in 24-well plates (Costar, Corning) and the cultures incubated at $34^{\circ} \mathrm{C}$ (Keck et al., 2000) in a $5 \% \mathrm{CO}_{2}$-enriched atmosphere.

\section{In Vitro Biofilm Model}

An in-house developed abiotic biofilm model mimicking long term-colonization was used. Strains were first grown in $\mathrm{mCDM}$ in planktonic growth conditions until mid-exponential phase. A 1:500 dilution of each strain of interest, corresponding to $10^{5}$ cells in $2.5 \mathrm{~mL}$ of $\mathrm{mCDM}$, was transferred to 24 -well plates and incubated at $34^{\circ} \mathrm{C}$ in $5 \% \mathrm{CO}_{2}$ for 72 hours. At $24 \mathrm{~h}$ and $48 \mathrm{~h}$, $2.0 \mathrm{~mL}$ of spent medium were carefully removed and $2.0 \mathrm{~mL}$ of pre-warmed fresh medium was added avoiding disturbance of the biofilm attached to the bottom of the well. At 72h, biofilms were resuspended in $300 \mu \mathrm{L}$ of PBS and either serially diluted for colony forming units (CFU) counts or sonicated to separate aggregates prior to flow cytometry analysis (described below). Sonication was done in a Bioruptor UCD-300 apparatus with an ultrasonic wave frequency of $20 \mathrm{KHz}(12$ cycles of 10 s at high intensity with 30 s break between pulses).

\section{Single-Strain and Dual-Strain Biofilm}

To evaluate interactions between strains, dual-strain biofilms were grown in a 1:1 ratio in the conditions described above. To allow for strain discrimination, dual-strain biofilm experiments used one GFP-labelled strain and one RFP-labelled strain. As a control, each pair of strains was also tested with the reverse fluorescent markers. For comparative analysis, single-strain biofilms of the same strain variants were obtained in parallel. For all experiments three biological replicates were done and each included three technical replicates.

\section{Flow Cytometry for Assessment of Cell Counts and Cell Density in Single-Strain and Dual-Strain Biofilm}

Cell counts of single- and dual-strain biofilms were obtained by flow cytometry as a proxy for cell viability. Biofilms disrupted by sonication (see above) were diluted in an appropriate volume of PBS to achieve a rate of 500 events/s. Cell counts were obtained in a Bio-Rad S3e Cell Sorter controlled by the ProSort software v1.6 using the following parameters: nozzle size of $100 \mathrm{microns}$, lasers of $488 \mathrm{~nm}$ and $561 \mathrm{~nm}$, and filters FL1 (525/30nm) and FL3 $(615 / 25 \mathrm{~nm})$ for data collection of GFP- and RFP-labelled cells, respectively. Flow cytometry conditions were set as: FSC $311 \mathrm{~V}$, SSC 240V, FL1 811V and FL3 335V. As the detection limit of the Bio-Rad S3e Cell Sorter is above the expression levels of RFPlabelled cells, these were detected as non-labelled in the FL1 filter.

For each pair of strains tested in dual-strain biofilm, the corresponding single-strain biofilms were first analyzed. Cells in single biofilms were counted (about 30000 events). Cells of the RFP-labelled strain were used to establish a "red region" in the FL1 Area log plot. Similarly, cells of the GFP-labelled strain were used to establish a "green region" in the same FL1 Area log plot. Potential overlap between these two regions was investigated and, when occurring, the relative proportion of GFP-labelled cells present in the "red region" was determined. For dual-strain biofilms, the relative proportion of the RFP-labelled and GFPlabelled strains was estimated by counting 30000 events and determining how many occurred in the red or green regions. When appropriate, a correction (based on the single-strain biofilm results) was applied. Finally, to estimate the cell density for each strain in single and dual-strain biofilms, total cell counts in $50 \mu \mathrm{L}$ were obtained.

\section{Identification of Intra-Species Interactions}

To investigate whether intra-species interactions occurred in the dual-strain biofilm experiments results obtained for a given pair of strains were combined in order to increase statistical power. For each strain, total cell counts obtained in single-strain biofilms (GFP- plus RFP-labelled variant cells) were compared to the total cell counts obtained for that strain in dual-strain biofilms. Interactions were considered to occur when statistically significant differences were observed $(\mathrm{p}<0.05)$. Upon detection of an interaction, a ratio between the geometric means of cell counts in dual- and single-strain biofilms was calculated. Negative interactions were defined by ratios $<1$ and positive interactions were defined by ratios $>1$.

\section{Confocal Microscopy for Biofilm Imaging}

Biofilms were grown in 24-well microscope slides suitable for confocal microscopy (IBIDI, Germany) as described above. Zsections were acquired at $0.81 \mu \mathrm{m}$ intervals on a Zeiss LSM 880 point scanning confocal microscope using the Airyscan detector, a 20x plan-apochromat 0.8 NA objective (Zeiss) and the $488 \mathrm{~nm}$ and $561 \mathrm{~nm}$ laser lines. The Zeiss Zen 3.0 (black edition) software was used to control the microscope, adjust spectral detection for the emission of GFP and RFP and to process the Airyscan raw images.

\section{Colorimetric Quantification of Biofilm Biomass Through Crystal Violet Staining}

Single- and dual-strain biofilms were grown for $72 \mathrm{~h}$ as described. At hour 72, supernatants were removed and biofilms were imaged before and after crystal violet staining. For staining, $50 \mu \mathrm{L}$ of crystal violet $(\mathrm{CV}, 1 \% \mathrm{w} / \mathrm{v})$ were added to each well and incubated at room temperature for $30 \mathrm{~min}$. CV was removed by inversion, the plates were allowed to air-dry for $10 \mathrm{~min}$ and the biofilms were washed twice with $500 \mu \mathrm{L}$ of PBS. Biofilms were 
imaged on a Zeiss Axio Zoom.V16 stereo microscope equipped with a Zeiss Axiocam 503 mono CCD camera and controlled with the Zen 2.1 software (blue edition), using the $1 \mathrm{X} 0.25 \mathrm{NA}$ objective and bright field optics. CV was solubilized by addition of $1 \mathrm{~mL}$ of $95 \%$ ethanol and incubation with agitation until no stained culture was observed at the bottom of the well. Absorbance at $595 \mathrm{~nm}$ was measured with a Tecan Infinite F200 plate reader. Absorbance was measured for three biofilms per strain or combination of strains.

\section{Statistical Analysis}

GraphPad Prism 7.0 (GraphPad Software Inc., La Jolla, California, USA) was used for all statistical analyses. The Kruskall-Wallis test corrected for FDR with the Benjamini and Hochberg method was used for comparisons between three groups. The Mann-Whitney U test was used for comparisons between two groups. The Mann-Whitney $U$ test corrected for FDR with the Benjamini and Hochberg method was used for the remaining comparisons.

\section{RESULTS}

\section{Fluorescence Labelling of Epidemiologically Relevant S. pneumoniae Strains}

We were able to successfully obtain GFP- and RFP-labelled variants of 14 pneumococcal strains of nine serotypes (serotypes 2, 3, 4, 15A, 19A, 19F, 22F, 33F, and NT) and 12 genetic backgrounds (STs 63, $177,205,260,276,344,433,595,717,4583,8322$, and 9161) (Table S2). Fluorescence microscopy allowed confirmation of GFP or RFP expression in all cells of the population for each fluorescent variant (Figures 1A, S1). The exceptions to this scenario were the RFPlabelled variant of strain 5262.1-19A and the GFP-labelled variant of strain 2099-3 in which fluorescence was noted in a small fraction of the population only (Figure S1, see, for example, cells marked with arrow).

We were unable, however, to transform eight other strains initially selected to be included in the study. These strains had the serotype-ST combinations 1-228, 11A-408, 15A-63, 19F-309, 33F-717, 35B-198, NT-3097, and NT-6996. Transformation of these strains was attempted multiple times using planktonic and biofilm conditions. Possibly, the laboratory conditions may have been inadequate to induce competence in these strains or the strains might have defects in genes essential for competence (Lee and Morrison, 1999; Croucher et al., 2011). Other studies have also reported difficulties in transforming non-laboratory strains (Joloba et al., 2010; Evans and Rozen, 2013).

\section{Cell Viability and Population Homogeneity of S. pneumoniae GFP- and RFP-Labelled Variants}

In preparation for dual-strain biofilm experiments mixing GFPand RFP-labelled strains, we investigated, for each labelled variant, the cell viability and the homogeneity of the fluorescence signal in $72 \mathrm{~h}$ biofilms.
Single strain biofilms were prepared and cell viability $(\mathrm{CFU} / \mathrm{mL})$ of the WT (unlabelled) strain and the corresponding GFP- or RFPlabelled variants was compared. Strains displayed different abilities to grow in biofilm, with mean $\mathrm{CFU}$ counts at $72 \mathrm{~h}$ ranging between $10^{5}$ and $10^{11} \mathrm{CFU} / \mathrm{mL}$ ( $\mathrm{p}<0.001$, Kruskal-Wallis test with Benjamini and Hochberg correction for False Discovery Rate). For most triplets (9 of 14) no significant differences were observed (Figures 1B, S1). One triplet (corresponding to WT strain 5756) showed significant differences in the viability of labelled variants (GFP vs RFP), but no significant differences between each variant and the WT (Figure 1B). The other four triplets (corresponding to WT strains 2099, 5262.2, 5902, and 8046) showed significant differences in cell viability (in at least one variant when compared to the WT strain) and were excluded from further analyses (Figure S1).

We then wanted to identify GFP and RFP variants yielding homogenous (narrow single peaks) and non-overlapping profiles, an essential requirement to enable cell counts of two populations of the same species by flow cytometry. With that in mind, the distribution of the fluorescence signal in the population was investigated by flow cytometry, for each strain variant (GFP or RFP), after growth of single-strain biofilms. For most variants, biofilm cells produced a homogenous signal (Figures 1C, S1). For two strains (5262.1 and 8276), however, high heterogeneity was observed for both the GFP and RFP signals (Figure S1). As this would compromise the capacity to distinguish mixed GFP- and RFP-producing cultures, these variants were excluded.

In summary, we selected GFP- and RFP- variants of eight strains to be used in dual-strain biofilm experiments.

\section{Dual-Strain Biofilm Experiments}

The eight strains remaining in the study were grown in dualstrain biofilms in which one strain was labelled with GFP and the other with RFP (for example, strain A-GFP was grown together with strain B-RFP), for a total of 28 combinations. As controls, the reverse labelling combination was also tested in a dual-strain biofilm (A-RFP together with B-GFP), and single-strain biofilms of all variants were also examined (A-GFP, A-RFP, B-GFP, and B-RFP) (Figure 2). In all cases, cell counts for each strain were measured at $72 \mathrm{~h}$ by flow cytometry.

For most combinations (21 out of 28), no significant differences in cell counts were observed when the isogenic GFP-and RFP-variants of a given strain were compared in the same experimental condition (Figures S2A-D). In seven combinations significant variations were observed in at least one comparison, in which case the assays were excluded from further analyses (Figure S2E).

In total, twenty-one combinations of dual-strain biofilms were validated for the investigation of intra-species interactions. These included strains of serotypes 2, 4, 15A, 19F, 22F, and NT of six distinct MLST profiles (Table 2 and Figure S2).

\section{Identification of Intra-Species Interactions}

To identify intra-species interactions, cell counts of each strain grown in dual-strain biofilm were compared to those obtained in a single-strain biofilm experiment run in parallel. Among the 21 
A

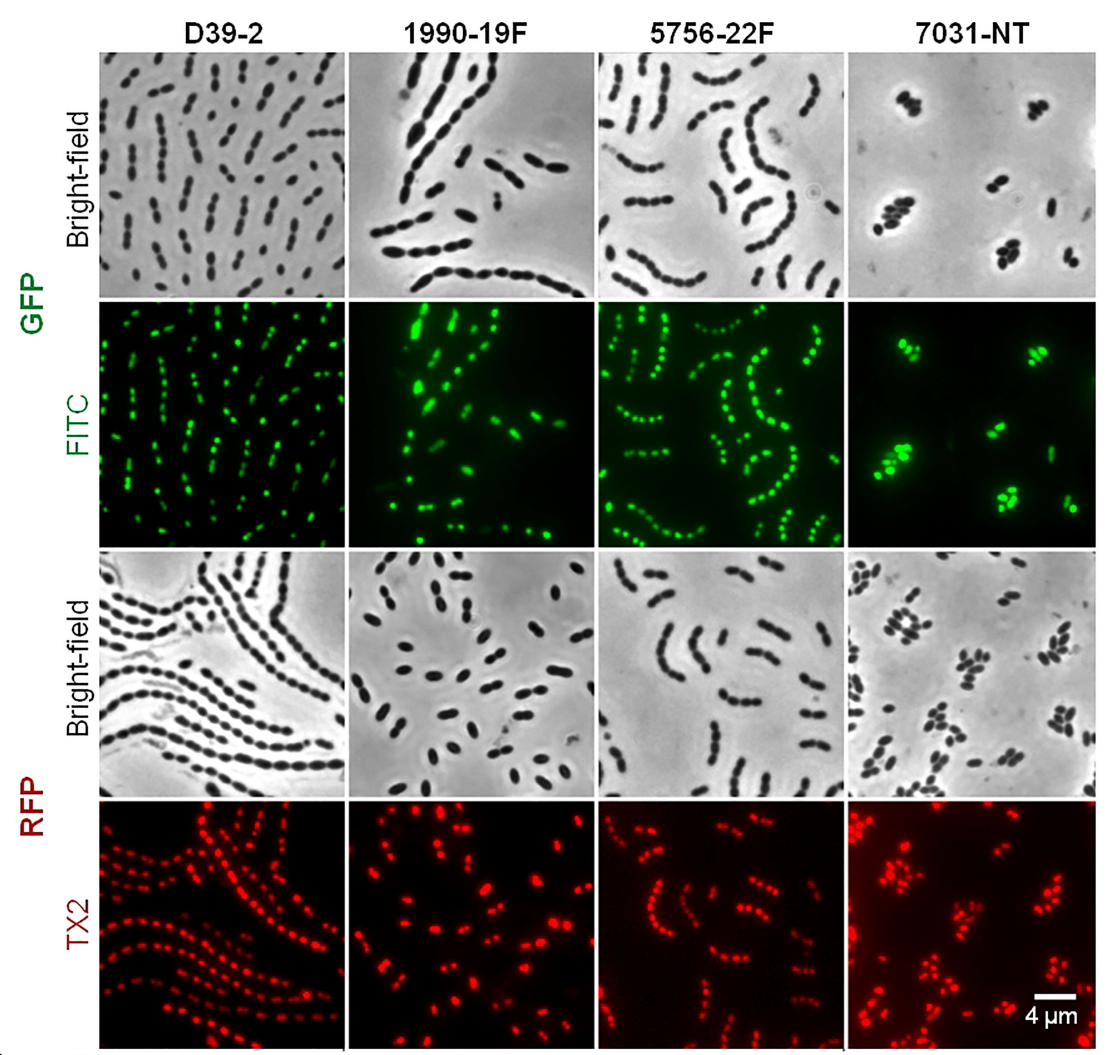

B

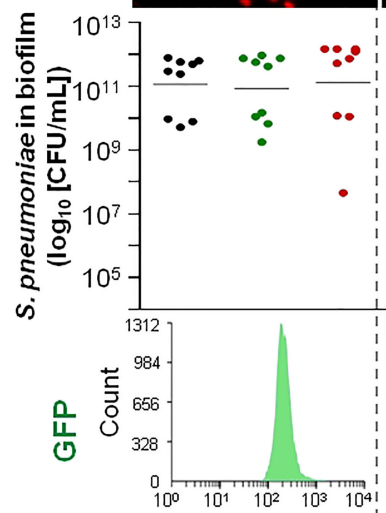

c

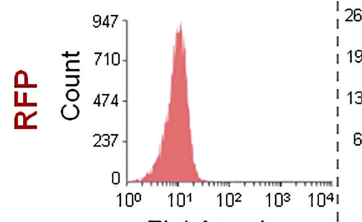

FL1 Area Log
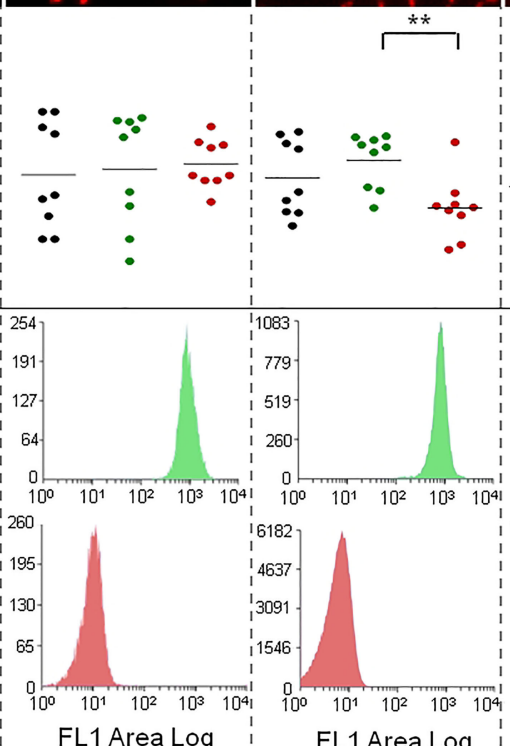

FL1 Area Log

FL1 Area Log

FL1 Area Log

FIGURE 1 | Representative fluorescently labelled strains used in the study (remaining strains are shown in Figure S1). Strains' reference and serotype are indicated on top of panel (A). (A) Imaging of GFP and RFP-labelled strains by fluorescence microscopy. GFP- and RFP-labelled strains were grown until exponential phase and imaged by fluorescence microscopy with filters for contrast phase optics, FITC and TX2. (B) Viability of wild-type and fluorescently labelled variants in the biofilm model. Each WT strain and its correspondent GFP- and RFP-labelled variants were grown under biofilm-promoting conditions (see the Materials and Methods section), after which viability (CFU/mL) was determined. Black, green and red dots indicate WT, GFP- and RFP-labelled cells, respectively. three independent experiments, each with an intra-experiment triplicate. ${ }^{\star *} \mathrm{p}<0.01$ (Kruskal Wallis test with Benjamini and Hochberg correction for FDR). (C) Flow cytometry analysis of fluorescent variants in biofilm model. Biofilm formation was induced as described in (B). After $72 \mathrm{~h}$ biofilms were resuspended and sonicated and cell counts were obtained by flow cytometry. Representative histograms are shown. 


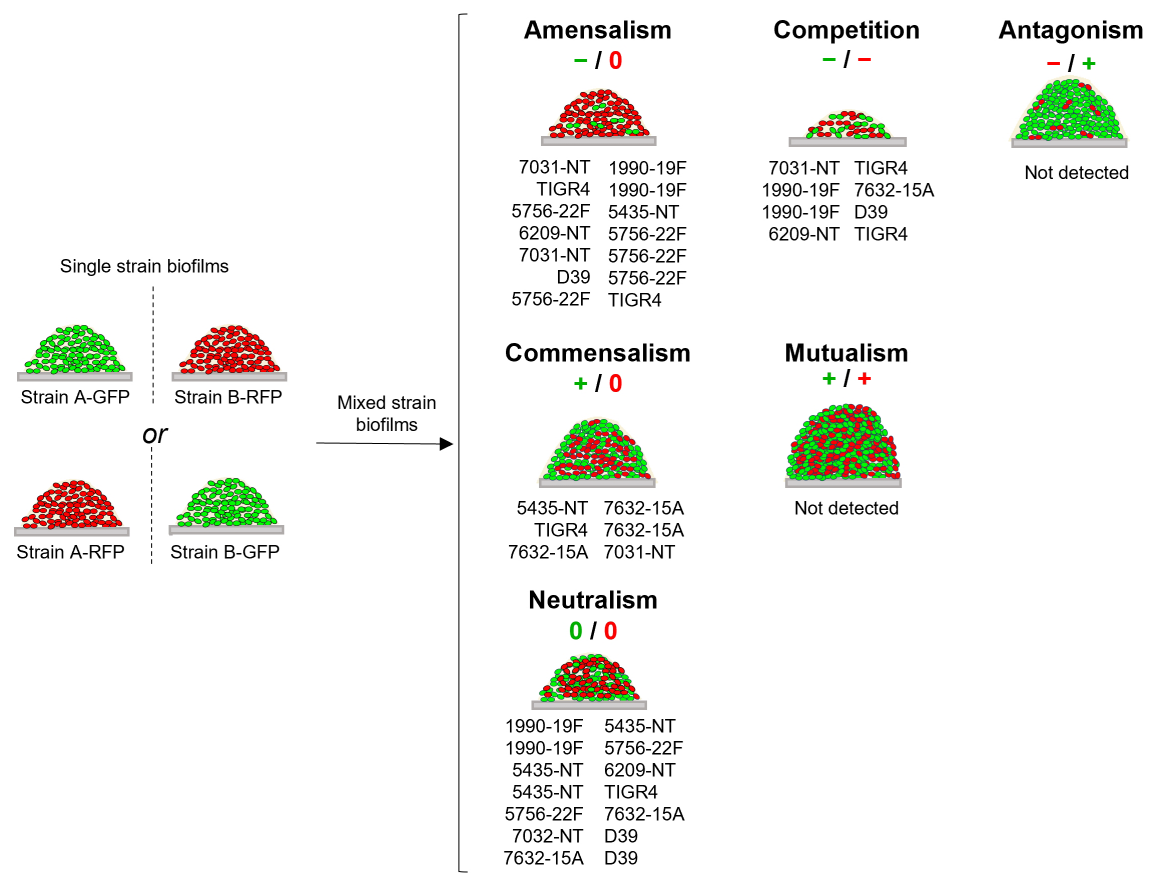

FIGURE 2 | Schematic representation of interactions identified among pneumococcal strains. For each strain growing in a dual-strain biofilm three possible effects can be observed when its cell counts are compared to those obtained when growing alone (single-strain biofilms depicted on the left): negative (-), if there is a decrease in its cell counts; positive (+), if there is an increase in its cell counts; and neutral $(0)$, if there is no change. Depending on the combination of effects observed for each pair of strains, interactions can be divided into six categories: neutralism (0/0), mutualism (+/+), commensalism (+/0), antagonism (-/+), amensalism $(-/ 0)$ and competition (-/-) [reviewed in (Faust and Raes, 2012)]. The interaction observed for each pair of strains is indicated below the schematic representation of each interaction.

combinations of dual-strain biofilms tested, interactions of commensalism $(n=3)$, competition $(n=4)$, amensalism $(n=7)$ and neutralism $(n=7)$ were detected (Table 3 and Figure 2, S2).

Importantly, we found that the outcome of an interaction is independent of the capsular and sequence types of the strains involved. An example that illustrates this observation is the interaction of strain 7632-15A (ST8322) with strains 5435-NT and 7031-NT (both ST344). In the first case (7632-15A and $5435-\mathrm{NT}$ ) the NT strain is benefited by the presence of the $15 \mathrm{~A}$ strain, whereas in the second case (7632-15A and 7031-NT) it is the $15 \mathrm{~A}$ strain that is benefited by the presence of the NT strain.

\section{Imaging of Intra-Species Interactions Occurring in Dual-Strain Biofilms}

Dual-strain biofilms representative of interactions of commensalism, competition, and amensalism were selected for confocal microscopy. Imaging of single- and dual-strain biofilms produced by strains 5435NT and 7632-15A confirmed the commensality identified between these two strains by flow cytometry (Figure 3A). In particular, we confirmed that strain 5435-NT increased in biofilm depth (z-axis, proxy to biofilm biomass) when in co-culture with strain 7632-15A (Figure 3A and Table S3).

Similarly, the competitive interaction observed by flow cytometry for strains 7031-NT and TIGR4 was also detected in the confocal microscopy images (Figure 3B). A decrease in biofilm depth was noted for both strains when grown in dualstrain biofilms as compared to their growth in single-strain biofilm (Figure 3B and Table S3).

Finally, the amensalism identified between strains 1990-19F and 7031-NT by flow cytometry (Figure 3C) was also observed by confocal microscopy imaging of these two strains when grown in single- and dual-strain biofilms. The significant decrease in cell counts (quantified by flow cytometry) of strain 7031-NT when growing in the presence of strain 1990-19F, translated into a major reduction of the biofilm produced by this strain when in co-culture with the $19 \mathrm{~F}$ strain. In fact, the total biofilm biomass of the dualstrain biofilms of this pair was found to be mostly attributable to the growth of the 19F strain (Figure 3C and Table S3).

The same representative pairs were also imaged before and after crystal violet staining (Figure S3A) and the total biofilm biomass was quantified (Figure S3B). The biomass quantified following crystal violet staining correlated with the biomass results obtained using CLSM.

\section{Imaging of Intra-Species Interactions Occurring in Dual-Strain Biofilms Grown in Different Concentrations of Choline}

To rule out possible effects of the choline concentration used in our mCDM $(1 \mathrm{mg} / \mathrm{mL})$ on the activity of choline-binding proteins - important for pneumococcal growth in biofilm and 
TABLE 3 | Dual-strain combinations tested for intra-species interactions.

\begin{tabular}{|c|c|c|c|c|c|}
\hline Strains tested & $\begin{array}{l}\text { Cell counts in dual biofilm } \\
\text { (GM dual) }\end{array}$ & $\begin{array}{l}\text { Cell counts in single biofilm } \\
\text { (GM single) }\end{array}$ & $\frac{\text { GM dual }^{b}}{\text { GM single }}$ & Net result ${ }^{c}$ & Type of interaction \\
\hline \multicolumn{6}{|c|}{ 5435-NT and 7632-15A } \\
\hline $7632-15 A$ & $2.05 \times 10^{8}$ & $8.10 \times 10^{8}$ & Ns & $=$ & \\
\hline \multicolumn{6}{|c|}{ 7632-15A and TIGR4 } \\
\hline $7632-15 A$ & $4.44 \times 10^{6}$ & $7.26 \times 10^{6}$ & Ns & $=$ & “ \\
\hline 7031-NT & $1.13 \times 10^{7}$ & $1.86 \times 10^{5}$ & ns & $=$ & “ \\
\hline $7632-15 A$ & $2.97 \times 10^{8}$ & $1.07 \times 10^{8}$ & $2.77 \times 10^{0}$ & $\uparrow$ & \\
\hline \multicolumn{6}{|c|}{ 7031-NT and TIGR4 } \\
\hline 7031-NT & $3.08 \times 10^{4}$ & $1.03 \times 10^{6}$ & $3.00 \times 10^{-2}$ & $\downarrow$ & Competition \\
\hline TIGR4 & $5.51 \times 10^{4}$ & $2.73 \times 10^{5}$ & $2.02 \times 10^{-1}$ & $\downarrow$ & \\
\hline \multicolumn{6}{|c|}{$1990-19 \mathrm{~F}$ and $7632-15 \mathrm{~A}$} \\
\hline D39 & $1.83 \times 10^{4}$ & $2.53 \times 10^{5}$ & $7.22 \times 10^{-2}$ & $\downarrow$ & \\
\hline \multicolumn{6}{|c|}{ 6209-NT and TIGR4 } \\
\hline 6209-NT & $1.66 \times 10^{5}$ & $3.46 \times 10^{6}$ & $4.80 \times 10^{-2}$ & $\downarrow$ & “ \\
\hline TIGR4 & $5.07 \times 10^{5}$ & $4.49 \times 10^{6}$ & $1.13 \times 10^{-1}$ & $\downarrow$ & \\
\hline \multicolumn{6}{|c|}{ 1990-19F and 7031-NT } \\
\hline 1990-19F & $2.23 \times 10^{6}$ & $2.29 \times 10^{6}$ & ns & $=$ & Amensalism \\
\hline 7031-NT & $1.18 \times 10^{4}$ & $2.66 \times 10^{7}$ & $4.44 \times 10^{-4}$ & $\downarrow$ & \\
\hline \multicolumn{6}{|c|}{ 1990-19F and TIGR4 } \\
\hline $1990-19 F$ & $1.24 \times 10^{5}$ & $2.98 \times 10^{5}$ & ns & $=$ & " \\
\hline TIGR4 & $1.99 \times 10^{4}$ & $2.10 \times 10^{5}$ & $9.47 \times 10^{-2}$ & $\downarrow$ & \\
\hline \multicolumn{6}{|c|}{$5435-\mathrm{NT}$ and $5756-22 \mathrm{~F}$} \\
\hline 5435-NT & $7.05 \times 10^{6}$ & $8.23 \times 10^{6}$ & ns & $=$ & “ \\
\hline D39 & $4.61 \times 10^{5}$ & $1.07 \times 10^{6}$ & $4.31 \times 10^{-1}$ & $\downarrow$ & \\
\hline $5756-22 F$ and $T$ & & & & & \\
\hline $5756-22 F$ & $3.60 \times 10^{4}$ & $1.44 \times 10^{6}$ & $2.49 \times 10^{-2}$ & $\downarrow$ & “ \\
\hline TIGR4 & $1.57 \times 10^{5}$ & $2.28 \times 10^{5}$ & ns & $=$ & \\
\hline $1990-19 \mathrm{~F}$ and 5 & & & & & \\
\hline 1990-19F & $2.49 \times 10^{5}$ & $5.77 \times 10^{5}$ & ns & $=$ & Neutralism \\
\hline 5435-NT & $8.41 \times 10^{4}$ & $9.92 \times 10^{4}$ & ns & $=$ & \\
\hline $1990-19 F$ and 5 & & & & & \\
\hline $1990-19 F$ & $7.80 \times 10^{5}$ & $1.77 \times 10^{6}$ & ns & $=$ & “ \\
\hline $5756-22 F$ & $4.33 \times 10^{5}$ & $5.84 \times 10^{5}$ & ns & $=$ & \\
\hline $5435-\mathrm{NT}$ and 6 & & & & & \\
\hline 5435-NT & $1.13 \times 10^{5}$ & $4.83 \times 10^{5}$ & ns & $=$ & “ \\
\hline 6209-NT & $6.88 \times 10^{6}$ & $7.96 \times 10^{6}$ & ns & $=$ & \\
\hline 5435-NT and T & & & & & \\
\hline 5435-NT & $7.62 \times 10^{4}$ & $1.27 \times 10^{5}$ & ns & $=$ & “ \\
\hline TIGR4 & $7.40 \times 10^{4}$ & $6.79 \times 10^{5}$ & ns & $=$ & \\
\hline $5756-22 \mathrm{~F}$ and 7 & & & & & \\
\hline $5756-22 \mathrm{~F}$ & $4.84 \times 10^{6}$ & $3.55 \times 10^{6}$ & ns & $=$ & " \\
\hline $7632-15 A$ & $3.89 \times 10^{7}$ & $1.74 \times 10^{7}$ & ns & $=$ & \\
\hline 7031-NT and D & & & & & \\
\hline 7031-NT & $3.57 \times 10^{5}$ & $2.79 \times 10^{6}$ & ns & $=$ & $"$ \\
\hline D39 & $7.61 \times 10^{5}$ & $1.62 \times 10^{6}$ & ns & $=$ & \\
\hline
\end{tabular}


TABLE 3 | Continued

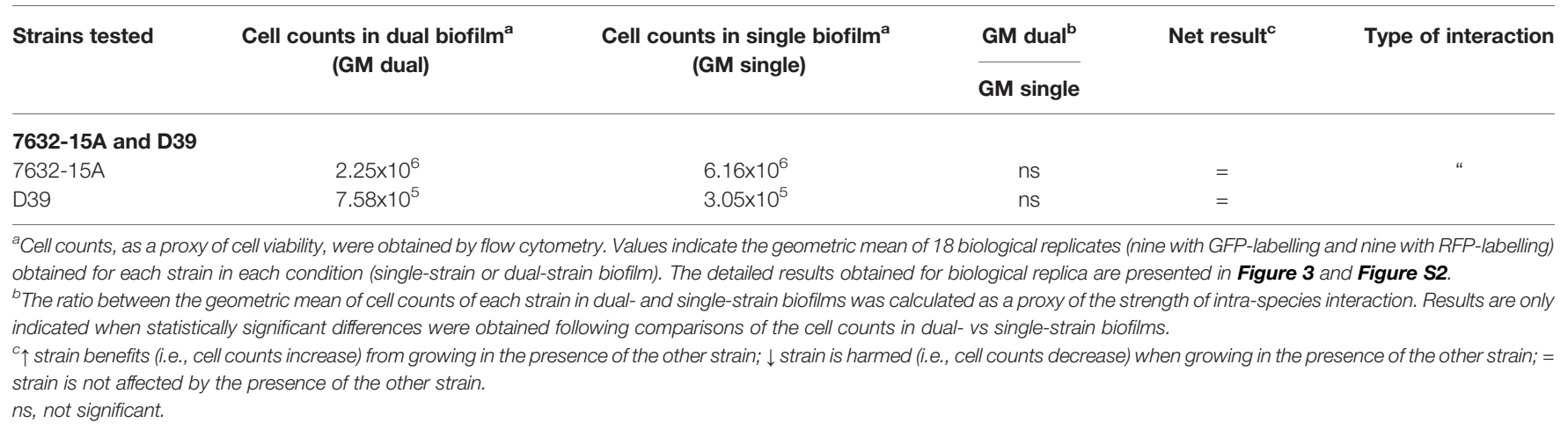

for fratricide (Moscoso et al., 2006; Perez-Dorado et al., 2010) -, single- and dual-strain biofilms representative of the interactions identified were grown in low $(4 \mu \mathrm{g} / \mathrm{mL})$ and high $(20 \mathrm{mg} / \mathrm{mL})$ concentrations of choline chloride.

At a lower concentration of choline $(4 \mu \mathrm{g} / \mathrm{mL})$ there was lower biofilm growth, both in single- and dual-strain biofilms. Still, the interactions previously observed were maintained (Figure S4). By contrast, the increase of choline chloride to $20 \mathrm{mg} / \mathrm{mL}$ severely hampered the ability of strains to grow in biofilm (in agreement with previous studies focusing on the importance of CBPs to biofilm development) (Moscoso et al., 2006). This lack of growth in biofilm prevented us to draw conclusions on the effect of this high concentration of choline in the interactions detected (Figure S5). Taken together, the results showed that the concentration of choline chloride used in our mCDM promotes better biofilm growth and has no impact on the interactions observed.

\section{DISCUSSION}

Multiple strains of pneumococci often coexist in the upper respiratory tract (Turner et al., 2011; Valente et al., 2012; Kamng'ona et al., 2015; Kandasamy et al., 2015; Valente et al., 2016b). The intra-species interactions resulting from this coexistence, however, have not been actively investigated. We developed a strategy to study such interactions and applied it to a diverse collection of $S$. pneumoniae strains.

We tested eight pneumococcal strains in pairs in a three-day long abiotic biofilm model. We found that, depending on the combination, strains could be positively affected, negatively affected or unaffected by the presence of other strain. Four types of interactions were observed: commensalism (14\%), competition (19\%), amensalism (33\%), and neutralism (33\%).

All positive interactions were of commensalism. To the best of our knowledge, this is the first report describing commensalism between naturally-occurring, colonizing strains of S. pneumoniae. We observed that strains of diverse backgrounds were positively affected, i.e. produced a better biofilm, in the presence of other strain.

Positive interactions between strains of the same species were identified before (Selak et al., 2016). Selak et al. showed that natural isolates of bifidobacteria obtained from intestinal biopsies cooperated for the degradation of complex carbohydrates. This resulted in cross-feeding and co-existence of strains with different metabolic profiles enabling colonization of diverse areas of the intestinal lumen (Selak et al., 2016). Since nutrient diffusion within biofilms is diminished (Stewart, 2003), it seems possible that the positive interactions identified in our study result, at least in part, from a mechanism of cross-feeding of metabolic by-products or nutrients resulting from cell lysis. Another possibility is that cooperation is occurring through the production of molecules that enable scavenging of otherwise unavailable nutrients or that degrade specific metabolites. We are conducting additional studies to address these possibilities.

Negative interactions were due to competition or amensalism. Both types of interactions were observed before in pneumococci. Pneumococcal colonization studies in children have repeatedly reported that, at any time, only a few serotypes account for most of the isolates, suggesting strain competition for niche occupancy affecting population structure and epidemiology (Félix et al., 2021). Biofilm studies and experiments with animal models have also unravelled these types of interactions and have explored some of the biological mechanisms governing them (Lipsitch et al., 2000; Dawid et al., 2007; Trzciński et al., 2015; Shen et al., 2019). Examples of such mechanisms include bacteriocin secretion (Dawid et al., 2007; Maricic et al., 2016) and exclusion of a newcomer by a resident strain via competencemediated fratricide (Shen et al., 2019). Mechanisms of passive competition, where one strain is indirectly affected by the presence of another, could also have a role in the negative interactions identified in our study. Differences in colonization ability attributable to the capsular type metabolic cost and associated cell surface charge (Lipsitch et al., 2000; Trzciński et al., 2015), in growth rates, or in tolerance to metabolic products or niche components, for example, could be involved.

Neutral interactions were detected in one third of the combinations tested indicating that either pneumococcal strains frequently co-exist without affecting each other (neutralism), or that those effects could not be detected with the system used. Neutral interactions among bacteria are not frequently described. Nevertheless, a recent study reported dominance of neutral interactions among actinobacteria in the soil ecosystem (Yan et al., 2021), particularly in oligotrophic conditions, i.e., in nutrient poor conditions that resulted from 
A
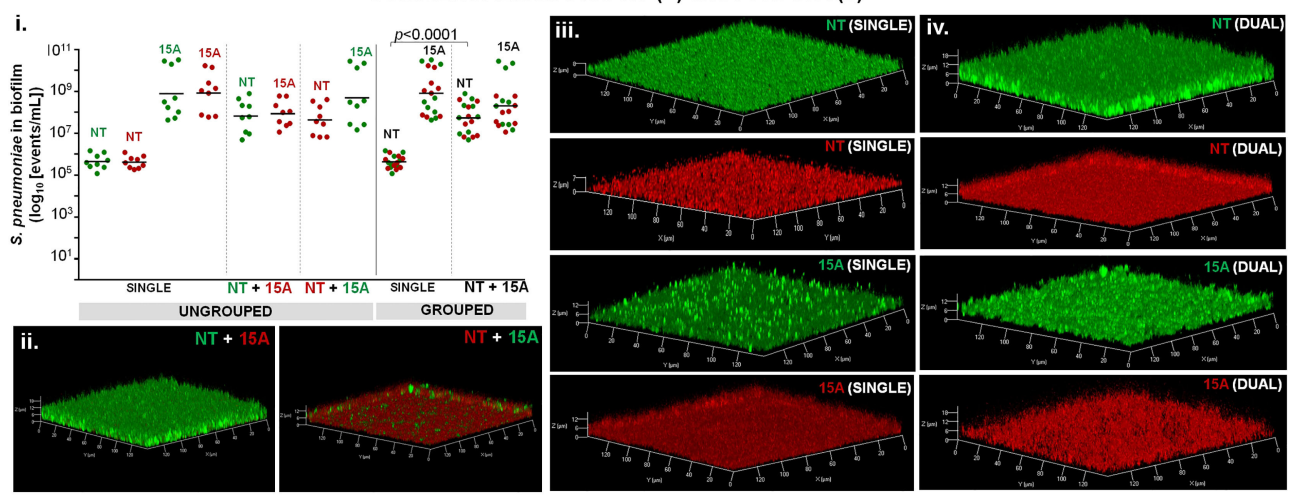

.
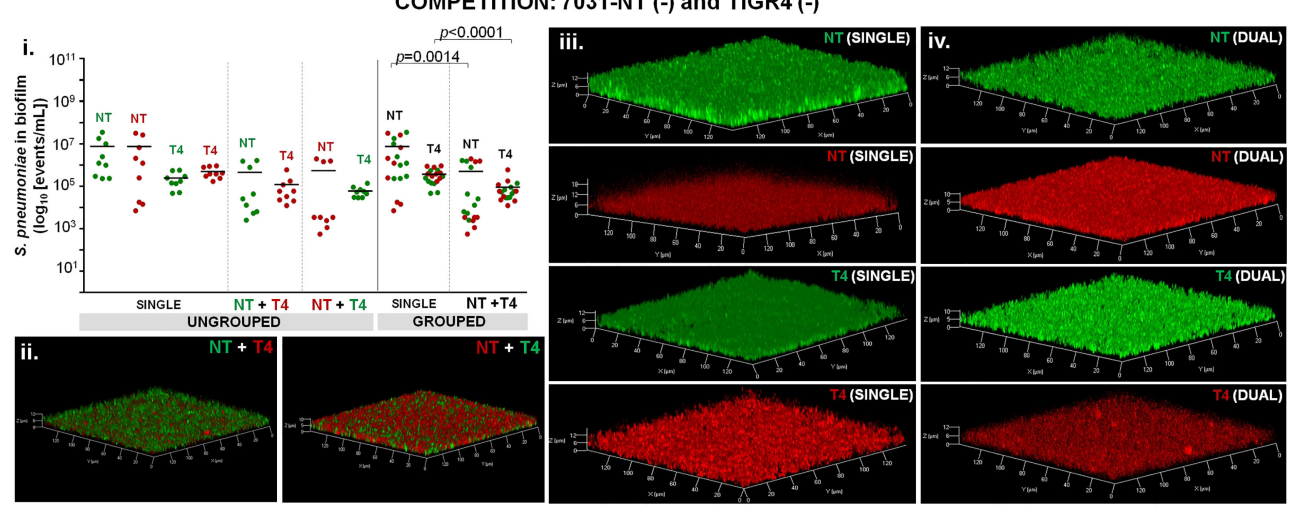

C
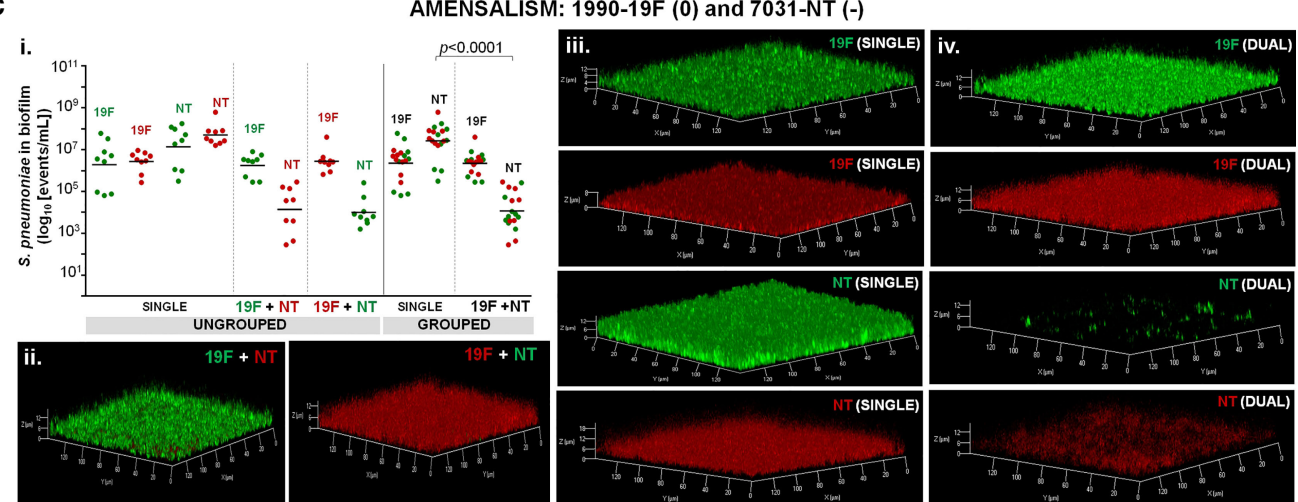

FIGURE 3 | Dual-strain combinations representative of commensalism (A), competition (B), and amensalism (C). Strains were tested in dual-strain biofilms (one GFP- and one RFP-labelled strain) in a 1:1 ratio. Reverse labelling combinations were also tested as controls. For comparative analysis, single-strain biofilms were grown in parallel. Intra-species interactions were considered to occur when statistically significant differences between a given strain in single and dual-strain biofilms were observed $(p<0.05)$, Mann-Whitney $U$ test corrected with Benjamini and Hochberg method for FDR. (i.) Cell counts. Cell counts of strains in single- and dualstrain biofilms were obtained by flow cytometry, events/mL were calculated and compared. For each strain, one out of three possible outcomes was determined when grown in the presence of another: positive (+), if there was beneficial effect; neutral (0), if there was no effect; and negative (-) if there was harm. Black bars indicate geometric means. Green and red dots correspond to GFP- and RFP-labelled cells, respectively. Graphics represent the ungrouped and grouped analyses for each pair using data from three independent experiments, each with an intra-experiment triplicate. (ii.) Imaging of dual-strain biofilms. (iii.) Imaging of single strain biofilms (iv.). Single-channel images of dual-strain biofilms. All CLSM images are transparent projections of 3D reconstructions from z-sections acquired at $0.81 \mu \mathrm{m}$ intervals on a Zeiss LSM 880 point scanning confocal microscope using the Airyscan detector, a 20x plan-apochromat 0.8 NA objective (Zeiss) and the 488 nm and $561 \mathrm{~nm}$ laser lines. 
metabolic overlap between species. Given the large array of metabolic profiles identified among pneumococcal strains (Watkins et al., 2015), it seems possible that our strains have different nutritional requirements; this possibility, however, was not addressed in the present study. It is also possible that intraspecies interactions may occur at a local level, with no impact on the total cells that form the biofilm (Kim et al., 2014). As populations of cells are being studied as a whole, such interactions would also have been missed with our approach.

Our study has some limitations. Firstly, our experimental approach was based on an in vitro system that does not take into consideration the effect that host- or microbiota-associated factors might have on pneumococcal intra-species interactions. In addition, our strains were inoculated in equal proportions and at the same time point. These are important limitations as, in natural colonization, pneumococcal strains co-occurring in the same host often are present at different proportions and may have been acquired in different occasions (Valente et al., 2012; Valente et al., 2016b). This approach prevented us from detecting positive or negative interactions that are dependent on relative cell-density and time of strains' exposure. An example where this scenario would be relevant is the competition model proposed by Shen and co-authors, in which earlier activation of the competence regulon, and hence the production of the fratricide effectors $\mathrm{CbpD}$ and $\mathrm{CibAB}$, conferred an advantage to the resident strain (occupying a niche) in the presence of a newcomer (Shen et al., 2019).

Secondly, some GFP- and RFP- labelled variants showed significant differences in cell viability compared to the parental strain; others had significant heterogeneity in the fluorescence signal. These limitations prevented the use of some strains with our experimental approach. Both fluorescent constructs used in this study were expressed under the control of the $h l p A$ promoter. Kjos et al. showed that for the laboratory strain D39, $h l p A-g f p$ and $h l p A-r f p$ fusions did not affect cell viability nor growth rate in liquid medium, were expressed throughout the cell cycle, and that fluorescence intensity remained high and stable with low cell-to-cell variation, as assessed by fluorescence microscopy (Kjos et al., 2015). Variation in the fluorescence signal, however, was not tested under biofilm-forming conditions, nor inspected for other strains or evaluated by flow cytometry. Possibly, the heterogeneity observed in our strains derives from natural variability previously described for isogenic strains (reviewed in (Jørgensen et al., 2013)), from the existence of a sub-population of cells that are either dying or dead but still retaining cell integrity (biofilms include many dead cells (Bayles, 2007)), or a combination of both. These limitations led to the exclusion of various strains limiting the magnitude of potential findings.

Thirdly, we observed heterogeneity within replicated biofilm experiments, which even though expected, hampered analyses of some dual-strain specific combinations. Marks et al. have shown that biofilms grown on an epithelial cell layer have increased reproducibility and lead to higher biofilm yields compared to biofilms obtained in abiotic conditions (Marks et al., 2012). In our optimized abiotic system, we have successfully obtained yields comparable to the ones described by Marks et al. The inclusion of an epithelial cell layer, albeit possibly increasing reproducibility, would increase the complexity of the analysis and would hinder accurate distinction and quantification of pneumococcal strains by flow cytometry.

In any event, the use of several strains led us to make two important observations: (i) the outcome of an interaction is independent of capsular and sequence type; (ii) a given strain, when grown in the presence of another, may be benefited, harmed or not affected, depending on the strain it is interacting with.

Overall, we were able to develop a strategy that enables identification of different types of intra-species interactions in biofilms and successfully applied it to a diverse collection of pneumococcal isolates. We validated the use of flow cytometry for direct quantification of bacteria within the biofilm, as opposed to more traditional methods, such as bacterial cell counts on selective media. Our approach enables discrimination between strains of the same serotype, genotype and antibiotype, overcoming a limitation of strategies previously described (Wu et al., 2017). Representative interactions were further confirmed through a qualitative approach based on CLSM, i.e., we were able to visualize positive, negative and neutral effects that $S$. pneumoniae strains may exert on each other and these were in agreement with the results obtained using flow cytometry. Furthermore, this approach could be adapted to other species and to more complex systems through the optimization of fluorescent constructs and the selection of other fluorescent reporters. In fact, a comparable strategy has recently been applied to visualize oral streptococcal species using integrative and replicative plasmids with strong promoters fused to fluorescent reporters (Shields et al., 2019).

In conclusion, we provide novel evidence that a panoply of intra-species interactions occur between naturally colonizing pneumococcal isolates and these are independent of serotype and genotype. These observations are a starting point to study the mechanisms underlying those interactions. Such studies will add to the growing body of knowledge regarding how the upper respiratory tract microbiota is shaped and adapts to changes triggered by the use of vaccines and antibiotics allowing the design of alternative strategies to modulate this ecosystem as a way to prevent infection.

\section{DATA AVAILABILITY STATEMENT}

The original contributions presented in the study are included in the article/Supplementary Material. Further inquiries can be directed to the corresponding author.

\section{AUTHOR CONTRIBUTIONS}

The study was designed by CV and RS-L. Data acquisition, analysis and interpretation were performed by $\mathrm{CV}, \mathrm{AC}, \mathrm{AH}$, and 
RS-L. The manuscript was drafted by CV, AC, and RS-L and critically revised by all authors. All authors contributed to the article and approved the submitted version.

\section{FUNDING}

This work was supported by project PTDC/BIA-MIC/30703/ 2017 from Fundação para a Ciência e a Tecnologia (FCT), Portugal; LISBOA-01-0145-FEDER-007660 (Microbiologia Molecular, Estrutural e Celular, funded by FEDER through COMPETE2020 - Programa Operacional Competitividade e Internacionalização); and LISBOA-01-0145-FEDER-016417 (ONEIDA co-funded by Fundos Europeus Estruturais e de Investimento, Programa Operacional Regional Lisboa 2020 and Fundação para a Ciência e a Tecnologia (FCT)). CV was supported by post-doctoral fellowship from FCT SFRH/BPD/ $115280 / 2016$. The funders had no role in the design of the study, collection, analysis, and interpretation of data, writing of the manuscript or in the decision to submit the manuscript for

\section{REFERENCES}

Avery, O. T., Macleod, C. M., and Mccarty, M. (1944). Studies on the Chemical Nature of the Substance Inducing Transformation of Pneumococcal Types : Induction of Transformation by a Desoxyribonucleic Acid Fraction Isolated From Pneumococcus Type Iii. J. Exp. Med. 79, 137-158. doi: 10.1084/jem.79.2.137

Azarian, T., Martinez, P. P., Arnold, B. J., Qiu, X., Grant, L. R., Corander, J., et al. (2020). Frequency-Dependent Selection can Forecast Evolution in Streptococcus Pneumoniae. PloS Biol. 18, e3000878. doi: 10.1371/journal.pbio.3000878

Barnes, D. M., Whittier, S., Gilligan, P. H., Soares, S., Tomasz, A., and Henderson, F. W. (1995). Transmission of Multidrug-Resistant Serotype 23F Streptococcus Pneumoniae in Group Day Care: Evidence Suggesting Capsular Transformation of the Resistant Strain In Vivo. J. Infect. Dis. 171, 890-896. doi: $10.1093 /$ infdis/171.4.890

Bayles, K. W. (2007). The Biological Role of Death and Lysis in Biofilm Development. Nat. Rev. Microbiol. 5, 721-726. doi: 10.1038/nrmicro1743

Blanchette, K. A., Shenoy, A. T., Milner, J.2nd, Gilley, R. P., Mcclure, E., Hinojosa, C. A., et al. (2016). Neuraminidase A-Exposed Galactose Promotes Streptococcus Pneumoniae Biofilm Formation During Colonization. Infect. Immun. 84, 2922-2932. doi: 10.1128/IAI.00277-16

Croucher, N. J., Harris, S. R., Fraser, C., Quail, M. A., Burton, J., van der Linden, M., et al. (2011). Rapid Pneumococcal Evolution in Response to Clinical Interventions. Science 331, 430-434. doi: 10.1126/science.1198545

Dawid, S., Roche, A. M., and Weiser, J. N. (2007). The Blp Bacteriocins of Streptococcus Pneumoniae Mediate Intraspecies Competition Both In Vitro and In Vivo. Infect. Immun. 75, 443-451. doi: 10.1128/IAI.01775-05

pt? >Evans, B. A., and Rozen, D. E. (2013). Significant Variation in Transformation Frequency in Streptococcus Pneumoniae. ISME J. 7, 791-799. doi: 10.1038/ ismej.2012.170

Faust, K., and Raes, J. (2012). Microbial Interactions: From Networks to Models. Nat. Rev. Microbiol. 10, 538-550. doi: 10.1038/nrmicro2832

Félix, S., Handem, S., Nunes, S., Paulo, A. C., Candeias, C., Valente, C., et al. (2021). Impact of Private Use of the 13-Valent Pneumococcal Conjugate Vaccine (PCV13) on Pneumococcal Carriage Among Portuguese Children Living in Urban and Rural Regions. Vaccine 39, 4524-4533. doi: 10.1016/ j.vaccine.2021.06.035

GBD (2018). Estimates of the Global, Regional, and National Morbidity, Mortality, and Aetiologies of Lower Respiratory Infections in 195 Countries 1990-2016: A Systematic Analysis for the Global Burden of Disease Study 2016. Lancet Infect. Dis. 18, 1191-1210. doi: 10.1016/S1473-3099(18)30310-4

Gray, B. M., Converse, G. M.3rd, and Dillon, H. C. Jr. (1980). Epidemiologic Studies of Streptococcus Pneumoniae in Infants: Acquisition, Carriage, and publication. The work was partially supported by PPBI Portuguese Platform of BioImaging (PPBI-POCI-01-0145FEDER-022122) co-funded by national funds from OE "Orçamento de Estado" and by European funds from FEDER "Fundo Europeu de Desenvolvimento Regional".

\section{ACKNOWLEDGMENTS}

The authors are grateful to Jan-Willem Veening for providing the fluorescent constructs for strain-labelling and to Ana Cristina Paulo for help with the statistical analysis.

\section{SUPPLEMENTARY MATERIAL}

The Supplementary Material for this article can be found online at: https://www.frontiersin.org/articles/10.3389/fcimb.2021. 803286/full\#supplementary-material

Infection During the First 24 Months of Life. J. Infect. Dis. 142, 923-933. doi: 10.1093/infdis/142.6.923

Hausdorff, W. P., Feikin, D. R., and Klugman, K. P. (2005). Epidemiological Differences Among Pneumococcal Serotypes. Lancet Infect. Dis. 5, 83-93. doi: 10.1016/S1473-3099(05)70083-9

Hiller, N. L., Ahmed, A., Powell, E., Martin, D. P., Eutsey, R., Earl, J., et al. (2010). Generation of Genic Diversity Among Streptococcus Pneumoniae Strains via Horizontal Gene Transfer During a Chronic Polyclonal Pediatric Infection. PloS Pathog. 6, e1001108. doi: 10.1371/journal.ppat.1001108

Horácio, A. N., Silva-Costa, C., Lopes, J. P., Ramirez, M., and Melo-Cristino, J. (2016). Serotype 3 Remains the Leading Cause of Invasive Pneumococcal Disease in Adults in Portugal, (2012-2014) Despite Continued Reductions in Other 13-Valent Conjugate Vaccine Serotypes. Front. Microbiol. 7, 1616. doi: 10.3389/fmicb.2016.01616

Isturiz, R., Sings, H. L., Hilton, B., Arguedas, A., Reinert, R. R., and Jodar, L. (2017). Streptococcus Pneumoniae Serotype 19A: Worldwide Epidemiology. Expert Rev. Vaccines 16, 1007-1027. doi: 10.1080/14760584.2017.1362339

Jørgensen, M. G., Van Raaphorst, R., and Veening, J. (2013). "Noise and Stochasticity in Gene Expression: A Pathogenic Fate Determinant," in Methods in Microbiology. Eds. C. Harwood and A. Wipat (London, UK: Academic Press), 157-175.

Joloba, M. L., Kidenya, B. R., Kateete, D. P., Katabazi, F. A., Muwanguzi, J. K., Asiimwe, B. B., et al. (2010). Comparison of Transformation Frequencies Among Selected Streptococcus Pneumoniae Serotypes. Int. J. Antimicrob. Agents 36, 124-128. doi: 10.1016/j.ijantimicag.2010.03.024

Junges, R., Salvadori, G., Shekhar, S., Amdal, H. A., Periselneris, J. N., Chen, T., et al. (2017). A Quorum-Sensing System That Regulates Streptococcus Pneumoniae Biofilm Formation and Surface Polysaccharide Production. Msphere 2, e00324-e00317. doi: 10.1128/mSphere.00324-17

Kamng'ona, A. W., Hinds, J., Bar-Zeev, N., Gould, K. A., Chaguza, C., Msefula, C., et al. (2015). High Multiple Carriage and Emergence of Streptococcus Pneumoniae Vaccine Serotype Variants in Malawian Children. BMC Infect. Dis. 15, 234. doi: 10.1186/s12879-015-0980-2

Kandasamy, R., Gurung, M., Thapa, A., Ndimah, S., Adhikari, N., Murdoch, D. R., et al. (2015). Multi-Serotype Pneumococcal Nasopharyngeal Carriage Prevalence in Vaccine Naive Nepalese Children, Assessed Using Molecular Serotyping. PloS One 10, e0114286. doi: 10.1371/journal.pone.0114286

Keck, T., Leiacker, R., Heinrich, A., Kuhnemann, S., and Rettinger, G. (2000). Humidity and Temperature Profile in the Nasal Cavity. Rhinology 38, 167-171.

Kim, W., Racimo, F., Schluter, J., Levy, S. B., and Foster, K. R. (2014). Importance of Positioning for Microbial Evolution. Proc. Natl. Acad. Sci. USA 111, E1639E1647. doi: 10.1073/pnas.1323632111 
Kjos, M., Aprianto, R., Fernandes, V. E., Andrew, P. W., Van Strijp, J. A., Nijland, R., et al. (2015). Bright Fluorescent Streptococcus Pneumoniae for Live-Cell Imaging of Host-Pathogen Interactions. J. Bacteriol. 197, 807-818. doi: 10.1128/JB.02221-14

Lee, M. S., and Morrison, D. A. (1999). Identification of a New Regulator in Streptococcus Pneumoniae Linking Quorum Sensing to Competence for Genetic Transformation. J. Bacteriol. 181, 5004-5016. doi: 10.1128/JB.181.16.5004-5016.1999

Lipsitch, M., Dykes, J. K., Johnson, S. E., Ades, E. W., King, J., Briles, D. E., et al. (2000). Competition Among Streptococcus Pneumoniae for Intranasal Colonization in a Mouse Model. Vaccine 18, 2895-2901. doi: 10.1016/S0264410X(00)00046-3

Maricic, N., Anderson, E. S., Opipari, A. E., Yu, E. A., and Dawid, S. (2016). Characterization of a Multipeptide Lantibiotic Locus in Streptococcus Pneumoniae. MBio 7, e01656-e01615. doi: 10.1128/mBio.01656-15

Marks, L. R., Reddinger, R. M., and Hakansson, A. P. (2012). High Levels of Genetic Recombination During Nasopharyngeal Carriage and Biofilm Formation in Streptococcus Pneumoniae. MBio 3, e00200-e00212. doi: 10.1128/mBio.00200-12

Moore, M. R., Link-Gelles, R., Schaffner, W., Lynfield, R., Lexau, C., Bennett, N. M., et al. (2015). Effect of Use of 13-Valent Pneumococcal Conjugate Vaccine in Children on Invasive Pneumococcal Disease in Children and Adults in the USA: Analysis of Multisite, Population-Based Surveillance. Lancet Infect. Dis. 15, 301-309. doi: 10.1016/S1473-3099(14)71081-3

Moscoso, M., Garcia, E., and Lopez, R. (2006). Biofilm Formation by Streptococcus Pneumoniae: Role of Choline, Extracellular DNA, and Capsular Polysaccharide in Microbial Accretion. J. Bacteriol. 188, 7785-7795. doi: 10.1128/JB.00673-06

Nunes, S., Felix, S., Valente, C., Simões, A. S., Tavares, D. A., Almeida, S. T., et al. (2016). The Impact of Private Use of PCV7 in 2009 and 2010 on Serotypes and Antimicrobial Resistance of Streptococcus Pneumoniae Carried by Young Children in Portugal: Comparison With Data Obtained Since 1996 Generating a 15-Year Study Prior to PCV13 Introduction. Vaccine 34, 16481656. doi: $10.1016 /$ j.vaccine.2016.02.045

Perez-Dorado, I., Gonzalez, A., Morales, M., Sanles, R., Striker, W., Vollmer, W., et al. (2010). Insights Into Pneumococcal Fratricide From the Crystal Structures of the Modular Killing Factor LytC. Nat. Struct. Mol. Biol. 17, 576-U572. doi: 10.1038/nsmb.1817

Rane, L., and Subbarow, Y. (1940). Nutritional Requirements of the Pneumococcus: I. Growth Factors for Types I, II, V, VII, VIII. J. Bacteriol. 40, 695-704. doi: 10.1128/jb.40.5.695-704.1940

Sá-Leão, R., Nunes, S., Brito-Avô, A., Frazão, N., Simões, A. S., Crisóstomo, M. I., et al. (2009). Changes in Pneumococcal Serotypes and Antibiotypes Carried by Vaccinated and Unvaccinated Day-Care Centre Attendees in Portugal, a Country With Widespread Use of the Seven-Valent Pneumococcal Conjugate Vaccine. Clin. Microbiol. Infect. 15, 1002-1007. doi: 10.1111/j.1469-0691.2009.02775.x

Sá-Leão, R., Pinto, F., Aguiar, S., Nunes, S., Carriço, J. A., Frazão, N., et al. (2011). Analysis of Invasiveness of Pneumococcal Serotypes and Clones Circulating in Portugal Before Widespread Use of Conjugate Vaccines Reveals Heterogeneous Behavior of Clones Expressing the Same Serotype. J. Clin. Microbiol. 49, 1369-1375. doi: 10.1128/JCM.01763-10

Selak, M., Riviere, A., Moens, F., Van Den Abbeele, P., Geirnaert, A., Rogelj, I., et al. (2016). Inulin-Type Fructan Fermentation by Bifidobacteria Depends on the Strain Rather Than the Species and Region in the Human Intestine. Appl. Microbiol. Biotechnol. 100, 4097-4107. doi: 10.1007/s00253-016-7351-9

Shen, P., Lees, J. A., Bee, G. C. W., Brown, S. P., and Weiser, J. N. (2019). Pneumococcal Quorum Sensing Drives an Asymmetric Owner-Intruder Competitive Strategy During Carriage via the Competence Regulon. Nat. Microbiol. 4, 198-208. doi: 10.1038/s41564-018-0314-4

Shields, R. C., Kaspar, J. R., Lee, K., Underhill, S. A. M., and Burne, R. A. (2019). Fluorescence Tools Adapted for Real-Time Monitoring of the Behaviors of Streptococcus Species. Appl. Environ. Microbiol. 85, e00620-e00619. doi: 10.1128/AEM.00620-19

Simões, A. S., Pereira, L., Nunes, S., Brito-Avô, A., De Lencastre, H., and Sá-Leão, R. (2011). Clonal Evolution Leading to Maintenance of Antibiotic Resistance Rates Among Colonizing Pneumococci in the PCV7 Era in Portugal. J. Clin. Microbiol. 49, 2810-2817. doi: 10.1128/JCM.00517-11

Stewart, P. S. (2003). Diffusion in Biofilms. J. Bacteriol. 185, 1485-1491. doi: 10.1128/JB.185.5.1485-1491.2003
Tettelin, H., Nelson, K. E., Paulsen, I. T., Eisen, J. A., Read, T. D., Peterson, S., et al. (2001). Complete Genome Sequence of a Virulent Isolate of Streptococcus Pneumoniae. Science 293, 498-506. doi: 10.1126/science.1061217

Tomasz, A. (1967). Choline in the Cell Wall of a Bacterium: Novel Type of Polymer-Linked Choline in Pneumococcus. Science 157, 694-697. doi: 10.1126/science.157.3789.694

Trzciński, K., Li, Y., Weinberger, D. M., Thompson, C. M., Cordy, D., Bessolo, A., et al. (2015). Effect of Serotype on Pneumococcal Competition in a Mouse Colonization Model. MBio 6, e00902-e00915. doi: 10.1128/mBio.00902-15

Turner, P., Hinds, J., Turner, C., Jankhot, A., Gould, K., Bentley, S., et al. (2011). Improved Detection of Nasopharyngeal Co-Colonization by Multiple Pneumococcal Serotypes Using Latex Agglutination or Molecular Serotyping by Microarray. J. Clin. Microbiol. 49, 1784-1789. doi: 10.1128/JCM.00157-11

Valente, C., Dawid, S., Pinto, F. R., Hinds, J., Simões, A. S., Gould, K. A., et al. (2016a). The Blp Locus of Streptococcus Pneumoniae Plays a Limited Role in the Selection of Which Strains can Co-Colonize the Human Nasopharynx. Appl. Environ. Microbiol. 82, 5206-5215. doi: 10.1128/AEM.01048-16

Valente, C., Hinds, J., Gould, K. A., Pinto, F. R., De Lencastre, H., and Sá-Leão, R. (2016b). Impact of the 13-Valent Pneumococcal Conjugate Vaccine on Streptococcus Pneumoniae Multiple Serotype Carriage. Vaccine 34, 40724078. doi: 10.1016/j.vaccine.2016.06.017

Valente, C., Hinds, J., Pinto, F., Brugger, S. D., Gould, K., Mühlemann, K., et al. (2012). Decrease in Pneumococcal Co-Colonization Following Vaccination With the Seven-Valent Pneumococcal Conjugate Vaccine. PloS One 7, e30235. doi: 10.1371/journal.pone.0030235

Valentino, M. D., Mcguire, A. M., Rosch, J. W., Bispo, P. J., Burnham, C., Sanfilippo, C. M., et al. (2014). Unencapsulated Streptococcus Pneumoniae From Conjunctivitis Encode Variant Traits and Belong to a Distinct Phylogenetic Cluster. Nat. Commun. 5, 5411. doi: 10.1038/ncomms6411

Waight, P. A., Andrews, N. J., Ladhani, S. N., Sheppard, C. L., Slack, M. P., and Miller, E. (2015). Effect of the 13-Valent Pneumococcal Conjugate Vaccine on Invasive Pneumococcal Disease in England and Wales 4 Years After its Introduction: An Observational Cohort Study. Lancet Infect. Dis. 15, 535543. doi: 10.1016/S1473-3099(15)70044-7

Watkins, E. R., Penman, B. S., Lourenço, J., Buckee, C. O., Maiden, M. C., and Gupta, S. (2015). Vaccination Drives Changes in Metabolic and Virulence Profiles of Streptococcus Pneumoniae. PloS Pathog. 11, e1005034. doi: 10.1371/ journal.ppat.1005034

Wei, H., and Havarstein, L. S. (2012). Fratricide is Essential for Efficient Gene Transfer Between Pneumococci in Biofilms. Appl. Environ. Microbiol. 78, 5897-5905. doi: 10.1128/AEM.01343-12

Weiser, J. N., Ferreira, D. M., and Paton, J. C. (2018). Streptococcus Pneumoniae: Transmission, Colonization and Invasion. Nat. Rev. Microbiol. 16, 355-367. doi: 10.1038/s41579-018-0001-8

Wholey, W. Y., Kochan, T. J., Storck, D. N., and Dawid, S. (2016). Coordinated Bacteriocin Expression and Competence in Streptococcus Pneumoniae Contributes to Genetic Adaptation Through Neighbor Predation. PloS Pathog. 12, e1005413. doi: 10.1371/journal.ppat.1005413

Wu, X., Jacobs, N. T., Bozio, C., Palm, P., Lattar, S. M., Hanke, C. R., et al. (2017). Competitive Dominance Within Biofilm Consortia Regulates the Relative Distribution of Pneumococcal Nasopharyngeal Density. Appl. Environ. Microbiol. 83, e00953-e00917. doi: 10.1128/AEM.00953-17

Wyllie, A. L., Chu, M. L., Schellens, M. H., Van Engelsdorp Gastelaars, J., Jansen, M. D., van der Ende, A., et al. (2014). Streptococcus Pneumoniae in Saliva of Dutch Primary School Children. PloS One 9, e102045. doi: 10.1371/ journal.pone. 0102045

Yan, B. F., Liu, N., Liu, M. H., Du, X. Y., Shang, F., and Huang, Y. (2021). Soil Actinobacteria Tend to Have Neutral Interactions With Other Co-Occurring Microorganisms, Especially Under Oligotrophic Conditions. Environ. Microbiol. 23, 4126-4140. doi: 10.1111/1462-2920.15483

Conflict of Interest: The authors declare that the research was conducted in the absence of any commercial or financial relationships that could be construed as a potential conflict of interest.

Publisher's Note: All claims expressed in this article are solely those of the authors and do not necessarily represent those of their affiliated organizations, or those of the publisher, the editors and the reviewers. Any product that may be evaluated in 
this article, or claim that may be made by its manufacturer, is not guaranteed or endorsed by the publisher.

Copyright $\odot 2022$ Valente, Cruz, Henriques and Sá-Leão. This is an open-access article distributed under the terms of the Creative Commons Attribution License
(CC BY). The use, distribution or reproduction in other forums is permitted, provided the original author(s) and the copyright owner(s) are credited and that the original publication in this journal is cited, in accordance with accepted academic practice. No use, distribution or reproduction is permitted which does not comply with these terms. 